\title{
Peter Derleder
}

\section{Die Eheleute Domrath \\ und die Vollstreckungsmacht der Banken beim Grundpfandkredit}

\begin{abstract}
Das deutsche Bankensystem hat den Nimbus anspruchsvollen, sorgfältigen Wirkens zugunsten spektakulärer Auftritte aufgegeben. Ein Bankensprecher fordert, dass die Kreditinstitute Refinanzierungszinsverbilligungen nicht an ibre Kreditkunden weitergeben. Es gebt nicht mebr um peanuts. Die Verstrickung in die Kreditverwendungsrisiken seit Anfang der goer Jabre hat zu dramatischen Verlusten gefübrt. Gibt es denn wenigstens Sektoren, wo von Solidität die Rede sein kann? Der erstrangige Grundpfandkredit scheint eine der Säulen zu sein, wo traditionelle Solidität herrscht. Der folgende Beitrag geht deswegen der Frage nach, ob das Funktionieren dieses Bereichs auf ausgewogenen Rechtsstrukturen oder auf einseitiger Machtbildung berubt.
\end{abstract}

\section{Einleitung}

Der sicherste Kredit ist der Grundpfandkredit. Vor allem für die Banken ist das richtig, wenn die Beleihung mit Sorgfalt erfolgt, die Grundstückswerte nicht aufgeblasen werden (wie im Zentrum von Tokio oder von Dr. Jürgen Schneider) und es dann auch bei Schuldnerkrisen nicht zu Ausfällen der Gläubigerbanken kommt, jedenfalls nicht beim erstrangigen Kredit bis zu einer Beleihungsgrenze von $60 \%$ des Grundstückswerts. $\mathrm{Zu}$ diesem Bodenkreditsystem gehört ferner der eherne Grundsatz, dass die Banken bei Zahlungsverzug oder Leistungsunfähigkeit der Schuldner diesen nicht hinterherlaufen müssen, möglicherweise in drei Gerichtsinstanzen zur Erlangung eines rechtskräftigen Titels. Deswegen bekommen sie von vornherein bei jeder Grundstücksbeleihung nach einer von ihnen in Jahrzehnten eingerichteten und von der Justiz nach und nach akzeptierten Rechtskonstruktion einen Titel in die Hand, regelmäßig eine vor einem Notar errichtete vollstreckbare Urkunde gem. $\$ 794$ Abs. I Nr. s ZPO, aus der sie bei jeder Unstimmigkeit mit dem Schuldner sofort vollstrecken können.

Das wird in einem ausgeklügelten System realisiert, in dem neben das Darlehen ein davon abstrahierendes Schuldanerkenntnis gem. $\$ 780 \mathrm{BGB}$ tritt, für das sich der Schuldner sogar der sofortigen Vollstreckung in sein persönliches Vermögen (also neben dem Grundstück) unterwerfen muss. Außerdem erhält die Bank in der Regel als Sicherheit eine Grundschuld über den Darlehensbetrag mit einer weit über die Darlehenszinsen hinausgehenden Hochzinsforderung, für die sich der Schuldner der sofortigen Zwangsvollstreckung in das Grundstück unterwirft. Und da niemand genau weiß, wie hoch das Darlehen bei einer späteren Leistungsstörung zwischen den Darlehensvertragsparteien valutieren wird, werden diese Verpflichtungen und Rechte auf dem Ausgangsstand eingefroren, also auf die Kapitalsumme ohne Rücksicht auf Tilgungsleistungen und zusätzlich auf den gesamten Zinsanspruch seit Darlehensauszahlung, unter Ignorierung der erbrachten Zinsleistungen. Da sich die Zinsen 
während der Darlehenslaufzeit erhöhen können, wird ein Maximalwert im Grundbuch fixiert, bei einem Darlehenszinssatz von 6\% pro Jahr also gern i $8 \%$ Zinsen auf die Grundschuld pro Jahr. Auch der Schuldner, der fünf Jahre Zins und Tilgung geleistet hat, schuldet ausweislich dieser Urkunden also neben der gesamten Summe des vollen Ausgangskapitals dann noch mehr als 90\% Zinsen darauf. Dieses für jeden unbefangenen Betrachter an sich monströs wirkende Schuldstatut ist die Basis eines jederzeitigen Vollstreckungszugriffs, mit dem die Bankkunden resolut zur Leistungstreue angehalten werden. Für den Vollstreckungsantrag ist es danach ohne Belang, ob der Schuldner Annuitäten nicht bezahlt hat, die Zinshöhe umstritten war oder eine unterschiedliche Verrechnung von Leistungen zum Dissens geführt hat. Das macht nichts, sagen die Banken mit Billigung der Justiz. Denn der Schuldner hat Gegenrechte. Er kann ja Klage erheben und seine Zahlungen nachweisen oder seine Rechnung und seine Rechtsansichten dem Gericht nachträglich plausibel machen. Ohne Anwalt wird er dies wegen der komplexen Rechtslage kaum können, die Klaginitiative liegt auch bei ihm, und Kosten hat er deswegen ebenfalls aufzubringen. Aber wenn er rechtzeitig für alles sorgt, kann er die Vollstreckung abwenden.

Immer wieder haben auch Zivilrechtler, die das Hohe Lied der Privatautonomie singen, auch für die Banken, Bedenken gegen diese Art einseitiger Machtstellung erhoben, da diese geradezu zum Missbrauch reizt. Sie haben allerdings nur sehr wenig zur Entschärfung beitragen können. Nach wie vor kann es zur Versteigerung eines Wohnhauses kommen, ohne dass je ein Gericht die Berechtigung der Bank und die Not des Häuslebauers geprüft hätte. Die Debatte ist so festgefahren, dass es eines lebendigen Beispiels für den hier verborgenen Unrechtsgehalt bedarf, bevor die rechtstheoretischen und -praktischen Konsequenzen erörtert werden können.

\section{Das lebendige Beispiel}

Karl Wilhelm Domrath aus Hilden in Westfalen ist heute 69 Jahre alt. Er ist zum Opfer dieses Grundpfandkreditsystems geworden und hat Haus und Hof verloren. Er ist zum Querulanten gestempelt worden, nachdem er sich umfassend gewehrt hat. Seine Sammlung unerträglich nichtssagender Bescheide, bei denen schon ein einziger substantieller inhaltlicher Satz hätte befreiend wirken können, könnte geradezu eine Skulptur alltäglicher Rechtsdefizite bilden. Eine Verfassungsbeschwerde aus seiner Feder hat auch nach zwei Jahren noch kein Aktenzeichen erhalten, abgesehen von den weißen Blättern, die ihm früher aus Karlsruhe zugeflattert sind. Siebzehn Jahre lang hat sich seine Rechtsangelegenheit zu einem Verfahrensdschungel ausgewachsen. Dabei war ihm dies keineswegs in die Wiege gelegt.

I934 in Bochum geboren, begann er zunächst eine Lehre im Bergbau, die jedoch wegen Kurzsichtigkeit abgebrochen werden musste. Nach einigen weiteren Tätigkeiten wurde er zum Industriekaufmann ausgebildet und besuchte danach eine höhere Wirtschaftsfachschule in Wilhelmshaven. Dort war er dann auch als Industriekaufmann bei den Olympia-Werken tätig, deren Schreibmaschinen heute nur noch romantische Vorstellungen auslösen. Er heiratete auch eine Wilhelmshavenerin und führte mit dieser eine glückliche Ehe, aus der eine Tochter hervorgegangen ist. Seine Ehefrau war Zahnarzthelferin und ab der Familiengründung Hausfrau. Mitte der 6oer Jahre bewarb sich Karl Wilhelm Domrath bei der Finanzverwaltung Nord-

I Die folgenden Sachverhaltsangaben sind, soweit möglich, aus Urkunden und Prozessakten der Darlehensschuldner rekonstruiert. 
rhein-Westfalen, wurde dann zum Steuerinspektor in Düsseldorf ausgebildet, kam in den Ministerialdienst des Landes Nordrhein-Westfalen und war in verschiedenen Ministerien tätig, so im Bauministerium, im Innenministerium und schließlich wieder im Städtebauministerium, zuletzt als Amtsrat. Viele Jahre lang war er ein solider, unauffälliger Beamter.

Bis Mitte der 8oer Jahre lebte er mit seiner Familie in einer Sozialwohnung als Mieter. Als er aufgrund seiner beruflichen Tätigkeit mit der in Sozialwohnungen geschuldeten Kostenmiete im Einzelnen befasst war, bemerkte er auch bei den Mietabrechnungen seiner Vermietungsgesellschaft Unkorrektheiten, die er rügte. Deswegen kam es zu seinem ersten Prozess, einem Mieterhöhungs- und Kündigungsprozess, den er hinsichtlich der Miethöhe zum Teil gewann, hinsichtlich der Kündigung aber schließlich beim Landgericht Düsseldorf rechtskräftig verlor. Es war dann auch kein Wunder, dass er einen Eigenheimerwerb anstrebte.

$\mathrm{Zu}$ diesem $Z$ weck schloß er zusammen mit seiner Ehefrau einen Bauträgervertrag über ein Reiheneckhaus mittlerer Preisklasse, bei dem er aber dem Angebot des Bauträgers entsprechend eine Reihe von Sonderwünschen verwirklicht haben wollte. Demgemäß wurde das Haus mit einem Erker versehen, das Wohnzimmer erhielt einen Parkettfußboden und einen Kamin, damit sollte der gemeinsame Sinn der Eheleute für ein gutes häuslichen Ambiente zum Ausdruck kommen.

Bei der Ausführung des Baus kam es zu einem Konflikt mit dem Bauträger, der mit der sachgerechten Erfüllung der Sonderwünsche zu tun hatte. Die Fertigstellung kam ins Stocken, der Bauträgervertrag wurde von beiden Seiten gekündigt. Die Stadtsparkasse Hilden, die die Finanzierung des Preises von 350.000 DM durch Vertrag vom 2. 5 . I 985 übernommen hatte, weigerte sich trotz vom Bauträger schriftlich bestätigten Baufortschritts, die fällige Rohbaurate von 3 I.000 DM auszuzahlen, obwohl sie sich dazu verpflichtet hatte. Maßgeblich dafür war ein zeitweiliger Baustillstand. Der Bauträger stand ohnehin auf der Kippe. Es kam dann zu einer Einigung über eine Zahlung von 3 I.000 DM zwischen den Bauherren, den Eheleuten Domrath, und dem Bauträger, der als Sicherheit eine Bankbürgschaft gab. Der Bauträger jedenfalls baute das Haus nicht weiter, sondern ging in Konkurs. Die Eheleute mussten mit einem neuen Architekten in eigener Regie das Haus zu Ende bauen. Von den im Darlehen zugesagten 350.000 DM hatte die Sparkasse nur 230.000 DM ausgezahlt. Dafür stand eine Grundschuld als Sicherheit zur Verfügung, mit der nicht nur das bereits auf die Eheleute umgeschriebene Hausgrundstück, sondern auch ein der Ehefrau gehörendes, weiteres unbelastetes Grundstück belastet wurden. Das gesicherte Darlehen war zu 6\% pro Jahr zu verzinsen, wobei die Zinsbindung auf fünf Jahre festgeschrieben war. In Wahrheit war der Zins, wirtschaftlich gesehen, wesentlich höher, da der Darlehensschuldner nur etwa 90\% der Darlehenssumme ausgezahlt erhalten hatte, der Rest also als Disagio verbucht war.

Da die Stadtsparkasse Hilden also von den versprochenen 350.000 DM nur 230.000 DM planmäßig ausgekehrt hatte, wegen der Finanzierung der weiteren Fertigstellung aber der Dissens über die Rohbaurate bestand, wollten die Eheleute Domrath, die auch gemeinsam Darlehensnehmer waren, das Kreditinstitut wechseln und gewannen für eine Umschuldung die Westdeutsche Landesbank, mit der sie einen Umschuldungsvertrag schlossen, dafür 2,5\% pro Jahr Bereitstellungszinsen bezahlten und deren Valuta zur Ablösung des Kredits bei der Stadtsparkasse Hilden bestimmt war. Aufgrund des Dissenses mit dieser kündigten die Kreditnehmer am 25.9. I 985 nach vier Monaten Laufzeit den Darlehensvertrag mit der Stadtsparkasse Hilden fristlos und stützten dies auf die verweigerte Zahlung der Rohbaurate. Für die Umschuldung sprachen auch wirtschaftliche Gründe, da die Kapitalmarktzinsen im Jahre i985 erheblich gesunken waren. 
Die Stadtsparkasse Hilden erkannte zwar die fristlose Kündigung nicht ausdrücklich an, nahm sie aber hin und verlangte eine Vorfälligkeitsentschädigung von 3.000 DM. Eine Vorfälligkeitsentschädigung für die vorzeitige Entlassung aus einem auf fünf Jahre fest geschlossenen Darlehensvertrag war in den Bedingungen des Darlehensvertrages nicht ausdrücklich vorgesehen; hier war lediglich von einer »Verwaltungskostenentschädigung «die Rede. Außerdem war kontrovers, ob die Darlehensnehmer auch das Disagio (in vollem Umfang) zurückzuzahlen hatten. Dieser Streitpunkt wurde jedoch später fallengelassen.

Von diesen umstrittenen 3.000 DM Vorfälligkeitsentschädigung aus nahm das Unglück seinen Lauf. Domrath lehnte diese Vorfälligkeitsentschädigung entschieden ab und verwies die Sparkasse darauf, ihn zu verklagen. Außerdem bot er ihr die Rückzahlung der geschuldeten 230.000 DM an, die die Westdeutsche Landesbank für ihn bereit hielt. Die Stadtsparkasse Hilden nahm diesen Betrag jedoch nicht an, da sie eine Leistung einschließlich der Vorfälligkeitsentschädigung erwartete. Von diesem Zeitpunkt an zahlte Domrath jahrelang nur Zinsen an die Westdeutsche Landesbank, obwohl diese ihm die Darlehensvaluta nicht ausgekehrt hatte, nämlich in Höhe von 2,5\% Bereitstellungszinsen bis Ende 1989. An die Stadtsparkasse Hilden zahlte er dagegen keine Zinsen mehr und stützte sich hierfür auf $\ 30 \mathrm{I}$ BGB, nach dem ein Gläubiger, der sich im sog. Annahmeverzug befindet, keine Zinsen zu beanspruchen hat.

Damit begann die Vollstreckungsperiode. Am 2 I. 2. 1986 stellte die Sparkasse Antrag auf Anordnung der Zwangsversteigerung beim Amtsgericht Langenfeld aus der im Grundbuch eingetragenen Grundschuld von 350.000 DM nebst I 8\% Jahreszinsen. Hierfür stützte sie sich auf eine vollstreckbare Ausfertigung der notariellen Urkunde über das Gesamtdarlehen und die Grundschuld. Das Vollstreckungsgericht hatte zunächst Bedenken, weil sich die Vollstreckungsklausel noch gegen den Voreigentümer richtete, gab dann aber dem Antrag statt und ordnete die Zwangsversteigerung aus dem dinglichen Titel am 24. 2. I 986 und aus dem persönlichen Titel am I. 4. 1986 an. Die Beschwerden zum Landgericht und zum Oberlandesgericht Düsseldorf in den Jahren I 986 und I 987 waren erfolglos, wobei beim OLG Düsseldorf ein Rechtsanwalt eingeschaltet war. Später legte Karl Wilhelm Domrath, inzwischen Experte seiner Rechtssache, seine Rechtsbehelfe ohne anwaltliche Beratung ein. In den abschlägigen Entscheidungen, insbesondere in einem Beschluss vom I8.4. 1986, wurde er darauf hingewiesen, dass er doch Vollstreckungsgegenklage gem. $\$ 767$ ZPO gegen die Titel erheben könne. Dies lehnte er jedoch ab, da er die Klägerrolle nicht übernehmen wollte und sich auf den Standpunkt stellte, eine nicht berechtigte Forderung auf Vorfälligkeitsentschädigung könne in keinem Fall die Zwangsversteigerung rechtfertigen. Er versuchte es auch mit anderen Rechtsbehelfen, insbesondere einer Erinnerung, die jedoch als unzulässig zurückgewiesen wurde.

$\mathrm{Zu}$ einer weiteren Zuspitzung kam es dann I988, als die Rechtspflegerin beim Amtsgericht Langenfeld die weitere Versteigerung durchzuführen in Angriff nahm. Wegen pointierter Formulierungen Domraths erstellte sie einen Vermerk, in dem sie seine Prozessfähigkeit in Zweifel zog und darauf verwies, dass er behauptet habe, ihm sei von der Justiz »eine Falle gestellt« worden. Demgemäß wurde die Sparkasse als Gläubigerin aufgefordert nachzuweisen, dass ihr Schuldner prozessfähig sei. Diese ließ sich nicht lumpen und verlangte lakonisch eine Pflegerbestellung. Daraufhin wurden die Eheleute zu einer Untersuchung ihrer Prozessfähigkeit geladen, ignorierten diese jedoch. Auch eine »Einladung « des Amtsrichters, der auch als Vollstreckungsrichter in dieser Sache fungiert hatte und nunmehr als Vormundschaftsrichter agierte, ließen sie unbeachtet, da sie aus dessen an sich freundlichem Schreiben am Ende doch eine Drohung mit der Entmündigung entnahmen. Domrath wurde 
dann zu einem Gespräch mit dem Richter und einem von diesem herangezogenen auswärtigen Psychiater vorgeführt, das gut zwei Stunden dauerte. Es wurde erstmals eine zwangsweise Unterbringung erwogen. Der Psychiater wollte aufgrund dieses Gesprächs aber noch kein Urteil über die Prozessfähigkeit abgeben. So kam es zu einer vierwöchigen zwangsweisen Unterbringung in einer Heilanstalt, wo der vom Richter bestellte Sachverständige, der Psychiater Dr. W-H., am Ende ein ausführliches Gutachten über einen unheilbaren Querulantenwahn erstellte. Diesem Gutachten lag vor allem die tatsächliche Feststellung einer fehlenden Bereitschaft zu außergerichtlicher Einigung zugrunde. Die »conclusio« des Gutachtens war, Domrath sei krankheitsbedingt nicht in der Lage, das mit einer Hypothek belastete Reihenhaus zu erhalten bzw. »die mit dem Kredit im Zusammenhang stehenden finanziellen Belastungen zu tragen«. Der Text des Gutachtens weist immer wieder juristische Einsprengsel auf, ohne dass ersichtlich wäre, inwieweit der Psychiater die dafür notwendigen tatsächlichen und rechtlichen Kenntnisse erworben hatte. Er rügte jedenfalls mangelnde Fähigkeit zum Vergleich. Erstmals erfuhr Karl Wilhelm Domrath hier aus diesem Gutachten, dass die Sparkasse eventuell auf ihre Vorfälligkeitsentschädigung von 3.000 DM verzichten wolle. Dies ist jedoch auch später nicht geschehen.

Aufgrund des Gutachtens wurde dann vom Vormundschaftsgericht die Betreuung angeordnet. Mit einem Einwilligungsvorbehalt für alle rechtlichen Aktivitäten wegen der Finanzierung des Hauses wurde am 30.9. I 992 endgültig die Betreuung für fünf Jahre angeordnet. Der Aufgabenkreis des Betreuers war die Sorge für das unbewegliche Vermögen einschließlich der Finanzierung, für die Wahrung der Rechte im inzwischen eingeleiteten Zwangspensionierungsverfahren und für alle gerichtlichen Verfahren. Der erste eingesetzte Betreuer gab bald sein Amt auf. Ein Kontakt zum zweiten Betreuer wurde ebenfalls nicht hergestellt. Der Ehefrau wurde die Betreuung nur angedroht. Dazu kam es aber nicht, da die Initiative zu den rechtlichen Schritten nur vom Ehemanne komme. Die Ehefrau hatte jedoch ebenfalls bekundet, dass hier krasses Unrecht geschehe. Verfassungsbeschwerden gegen die Anordnung der Zwangsversteigerung und die Anordnung der Betreuung blieben erfolglos. Soweit einmal eine Begründung gegeben wurde, war diese formaler Art.

Im Jahre I 990 hatte Domrath sich wegen der Verstrickung in die Grundstücksangelegenheit krank gemeldet und dann auch Dienstbefreiung beantragt. Die Behörde leitete ein Zwangspensionierungsverfahren ein, bei dem zwei Gutachten eingeholt wurden, neben dem Gutachten des Dr. W-H. ein zweites kürzeres, das dem Erstgutachten ohne zusätzlichen Begründungsaufwand folgte. Die Zwangspensionierung mit 59 Jahren führte zu einer nicht unerheblichen Verkürzung der Pension.

In der Versteigerungssache kam es am 30. 7. 1992 zum ersten Termin, ohne dass zuvor über den Befangenheitsantrag gegen die zuständige Rechtspflegerin entschieden worden wäre. Der vor Gericht entmündigte Ehemann brachte seine Ehefrau mit, die das Meistgebot abgab und der ein Jahr später der Zuschlag für das gemeinsame Hausgrundstück erteilt wurde. Sie brachte den Grundschuldbetrag jedoch nicht auf. Es kam so zur Einleitung der Wiederversteigerung. Beim Termin vom 8. 9. I 994 wurde der Zuschlag einem Versteigerungsunternehmer erteilt, der für $404.000 \mathrm{DM}$ das Hausgrundstück erwarb. Bei seinem ersten Besuch sagte er zu den Eheleuten: »Sie haben es aber schön.« Deswegen nutzte er das Hausgrundstück später selbst. In der Folgezeit wurde das andere Grundstück, das der Ehefrau gehörte, für 36r.000 DM versteigert. Bei der Verteilung des Versteigerungserlöses erhielt die Stadtsparkasse Hilden alles, was sie beanspruchte, also das Kapital einschließlich Disagio, Vertragszinsen für die gesamte Zeit bis zur Befriedigung sowie weiteren Verzugsschadensersatz. Die Vorfälligkeitsentschädigung wurde nicht berechnet, da die Vertragslaufzeit von fünf Jahren längst abgelaufen war. 
Konsequenterweise kam es Ende 1994 zur Zwangsräumung. Die Eheleute, die sich keine Ersatzwohnung beschafft hatten, wurden mit dem Gerichtsvollzieher aus der Wohnung gewiesen. Sie kamen zunächst in einem Zimmer eines Stundenhotels unter und bezogen Anfang I995 dann eine Dreizimmermietwohnung. Ihr Mobiliar konnte nur teilweise in die Mietwohnung gebracht werden und blieb im Übrigen eingelagert. Mit dem Erlös wurde die Sparkasse in vollem Umfang befriedigt, jedoch unter Absehung von einer Vorfälligkeitsentschädigung. Es blieben noch ca. 270.000 DM übrig, die beim Amtsgericht Langenfeld seitdem hinterlegt sind. Die Eheleute wollten diesen Betrag nicht annehmen, da sie von einer unrechtmäßigen Enteignung ausgingen. Dagegen unternahmen sie weiterhin vielfältige rechtliche Schritte. Dazu gehörten außer den Verfassungsbeschwerden Petitionen und Eingaben. Die Adressaten waren einzelne Abgeordnete, der Ministerpräsident des Landes NordrheinWestfalen, die Landesregierung, der Bundespräsident, der Petitionsausschuss des Deutschen Bundestages, alles Institutionen, die mit keinem Wort auf das Vollstreckungssystem eingingen. Im vorvergangenen Winter ist die Ehefrau im Alter von 73 Jahren verstorben. Der Ehemann hegt ihr gegenüber Schuldgefühle, da sie nur seinetwegen die rechtlichen Schritte mitgetragen hat.

Karl Wilhelm Domrath hat sich die Materien des Darlehensrechts und des Vollstreckungsrechts im Laufe der Jahre bis ins Detail erarbeitet. Er hat die von den Banken benutzten und die als Alternative vorgeschlagenen Klauseln im Einzelnen untersucht, die Rechtsentwicklung zur Vollstreckungsgegenklage rezipiert und sich Vorstellungen über eine bessere Regelung gemacht, wobei er die rechtlichen Interessen der Banken an einer zügigen Inanspruchnahme der Grundstückswerte keineswegs verkennt. Er ist aber in den angrenzenden Rechtsgebieten, insbesondere auch des Verfassungsgerichtsprozesses, Laie geblieben, so dass eine Vielzahl seiner Rechtsbehelfe von vornherein aussichtslos war.

Er ist nach wie vor eine sehr sachliche Persönlichkeit mit freundlichem Diskurs. Er hat nie physisch eine Tür zugeschlagen oder einen Bankangestellten oder Richter persönlich beleidigt. Er hat sich im Zuge seiner Verfahren allerdings schriftlich auf zwei riskante Formulierungen festgelegt, nämlich dass es sich um eine »Fälschung « handle, soweit ein vollstreckbarer Titel gegen ihn zugrunde gelegt worden ist, und dass insgesamt ihm gegenüber eine »Rechtsbeugung « begangen worden sei. Damit hat er für die Anordnung der Betreuung einen Anlass gegeben. Diese Betreuung ist erst nach der Versteigerung der Grundstücke aufgehoben worden. Sie war zwar mit der Begründung angeordnet worden, dass er vor der Versteigerung des Grundstücks geschützt werden müsse, erwies sich dann aber als Mittel zur Durchsetzung der Vollstreckung. Die nichtssagenden Bescheide und Mitteilungen sind teilweise so substanzlos gefasst, dass ihre Lektüre Affekte provozieren muss. ${ }^{2}$

Es hat allerdings für diesen hartnäckigen Beamten aber auch niemals eine Person in der Justiz gegeben, die ihm als Mensch gegenübergetreten wäre und das sortiert hätte, was die herrschende Rechtsauffassung ist, was vertretbare Alternativen sind, was rechtlich und moralisch anstößig ist. Dagegen hat er sich gegenüber dem obstinaten Begehren von Justizautoritäten und Psychiatern als intransigent erwiesen, die ihm unter Ab-

2 Als Beispiel sei ein Schreiben des AG Langenberg vom 22. I I. 1996 an die Ehefrau Domrath zitiert: "Sehr geehrte Frau Domrath, in der Zwangsversteigerungssache Domrath hat der zuständige Abteilungsrichter von einer Verbescheidung der von Ihnen eingelegten Zuschlagsbeschwerde wegen Rechtsmissbrauchs abgesehen (vgl. auch Zeller/Stöber ZVG, I4. Aufl., Rn. 8.4 der Einleitung). Der Zuschlagsbeschluss ist damit rechtskräftig. Der Verteilungstermin am I I. I 2. I 996 wird wie geplant stattfinden...« Der Antrag einer prozessfähigen Partei kann zwar wegen Rechtsmissbrauchs möglicherweise auch in einem einzigen Satz durch Beschluss zurückgewiesen werden oder auch etwa mit dem Sachargument, dass das Vollstreckungsgericht zur Prüfung der erhobenen materiellrechtlichen Einwände nicht befugt sei. Mit der begründungslosen Versagung eines Beschlusses wird jedoch das Territorium des Rechtsstaates verlassen. 
sehen von allen rechtlichen Fragen aus lebenspraktischen Gründen das Einlenken

aufgedrängt haben. Das war gewiss keine Stärke, da oft auch die Verarbeitung von Unrecht eine lebenspraktische Notwendigkeit ist. Der geläufige Mechanismus, dass einer, der der Justiz zu oft kommt, zum Kohlhaas abgestempelt wird, hat bei ihm gegriffen, so dass auch die Gefahr besteht, dass sein radikaler Widerstand gegen das Vollstreckungszugriffssystem der Banken beim Grundpfandkredit ganz folgenlos bleibt. Er plant aber heute die Gründung einer Stiftung, die sich die Verwirklichung von Humanität im Vollstreckungs- und im Betreuungsverfahren zur Aufgabe macht. In jedem Fall zeigt das Schicksal der Eheleute Domrath mit letzter Konsequenz auf, wie viele Rechte die Banken als Grundpfandgläubiger für sich aufgetürmt haben.

\section{Das Unrecht gegenüber den Eheleuten Domrath}

Bevor über die grundsätzlichen rechtlichen Lehren aus diesem Fall nachgedacht werden kann, ist zunächst einmal der Versuch einer rechtlichen Bewertung zu unternehmen, wie sie notwendig gewesen wäre, wenn die Sparkasse ihre Ansprüche hätte begründen müssen. Dabei wird die schwer überschaubare Verbindung der verschiedenen Rechtsgebiete deutlich.

\section{Darlehensauszablung, Kündigung, Vorfälligkeitsentschädigung und Vertragsverletzung}

\section{a) Die unvollständige Darlehensauszablung}

Die Sparkasse schuldete den Darlehensnehmern Domrath vertraglich die Auszahlung von 350.000 DM. Die Auszahlung geschieht bei der Finanzierung von Bauträgerverträgen regelmäßig nicht voraussetzungslos, selbst wenn der Darlehensnehmer das Kreditinstitut zur Auszahlung an den Bauträger anweist. Denn das Kreditinstitut will regelmäßig nur entsprechend der Werterhöhung des als Sicherheit dienenden Baugrundstücks auszahlen und macht deswegen vertraglich meist die Auszahlung in Raten nach dem Baufortschritt zur Bedingung. Das ist auch sein gutes Recht, da es nur so eine der Valutierung entsprechende Sicherheit gewährleisten kann.

Kommt es nun zu einem Konflikt zwischen Bauträger und Bauherrn, etwa im Zuge der Verwirklichung von Sonderwünschen, der Verzögerung der Bauerstellung oder wegen mangelnder Leistungsbereitschaft oder -fähigkeit des Bauträgers, dann muss der Bauherr, der mit dem Kreditinstitut einen selbstständigen Darlehensvertrag geschlossen hat, grundsätzlich damit rechnen, dass auch die Auszahlung der Darlehensraten ins Stocken gerät und bei Einstellung der Bautätigkeit keine weiteren Raten ausbezahlt werden. Es ist die Sache des Bauherrn, mit dem Bauträger die planmäßige Bauerrichtung zu realisieren. Misslingt dies, so kann der Bauherr vom finanzierenden Kreditinstitut regelmäßig auch keine (weiteren) Kreditauszahlungen erwirken, da das Kreditinstitut sonst ohne hinreichende Sicherheit dastehen würde.

Im Streit um die Zahlung der Rohbaurate, die Verrechnung bereits separat aufgebrachter 3 I.000 DM, die Stockung des Baus sowie den Konkurs des Bauträgers konnte die Sparkasse nach dem Vertragstext mangels planmäßigen Baufortschritts eventuell die Auskehrung dieser Rate verweigern. Sie konnte zwar davon ausgehen, dass ein Bauherr normalerweise alle Mittel mobilisiert, um den angefangenen Bau zu voll- 
enden. Ein Kreditinstitut kann sich damit jedoch nicht beruhigen, sondern darf darauf bestehen, dass die Sicherheit in jedem Moment des Bauerstellungsprozesses für den Kredit ausreicht. Deswegen hätte die Verweigerung der Rohbaurate berechtigt sein können, wenn n u r das Baugrundstück als Sicherheit gedient hätte.

So war es aber gerade nicht, da die Sparkasse sich von vornherein eine Grundschuld an dem unbebauten Grundstück der Ehefrau hatte geben lassen, das später dann für 36r.000 DM versteigert wurde. Nun ist damit noch nicht geklärt, welchen Wert es zum Zeitpunkt der Grundschuldbestellung im Jahre i985 hatte. Denkbar wäre eine erhebliche Wertsteigerung während des Zeitraums zwischen Darlehensvertragsabschluss und Zuschlag, auch wenn das Grundstück stets unbebaut war. In jedem Fall war eine über den Wert des Baugrundstücks hinausgehende Sicherheit gewährt. Wenn aber ein Sicherungsdefizit der Sparkasse, gemessen an der üblichen Sicherung bei einem Grundstückserwerb, nicht vorhanden war, dann handelte die Sparkasse rechtsmissbräuchlich, wenn sie sich trotz überschießenden Sicherungswerts auf die Baufortschrittsklausel berief und die Zahlung einer Rate von 3 I.000 DM verweigerte. Damit hätte sie ohne ein erkennbares wirtschaftliches Interesse die planmäßige Vertragsdurchführung gestört. Dafür, dass es so war, spricht maßgeblich, dass die Sparkasse sich nur auf den Buchstaben ihrer Baufortschrittsklausel berufen, die zusätzliche Sicherheit überhaupt nicht berücksichtigt und auch kein Sicherungsdefizit behauptet hat.

\section{b) Die Kündigung des Kredits}

Nach dem Konkurs des Bauträgers mussten die Darlehensschuldner wegen der Verweigerung weiterer Darlehensauszahlung die Fertigstellung des Baus anderweit organisieren und hatten dies auch zu finanzieren. Eine unberechtigte Auszahlungsverweigerung durch die Sparkasse konnte einen wichtigen Grund analog $\$ 626$ BGB a. F. für eine vorzeitige fristlose Kündigung des fest auf fünf Jahre geschlossenen Darlehensvertrags darstellen. ${ }^{3}$ Hierfür kann eine vorherige Abmahnung erforderlich sein, wenn die weitere Darlehensauszahlung nicht bereits endgültig und ernsthaft verweigert worden ist. Auch musste eventuell die Zweiwochenfrist des $\ 626$ Abs. 2 BGB gewahrt werden. Ob all diese Wirksamkeitsvoraussetzungen vorlagen, lässt sich nach so vielen Jahren nicht mehr abschließend detailgenau feststellen. Keine Frage ist aber, dass ein Darlehensnehmer, dem zu Unrecht ein Teil der Darlehensvaluta vorenthalten wird, das Recht zur fristlosen Kündigung des gesamten Darlehens hat, da er dann die Gesamtfinanzierung nicht mehr planmäßig realisieren kann und sich notwendigerweise um einen anderen Kreditgeber bemühen muss.

\section{c) Der Anspruch auf Vorfälligkeitsentschädigung}

Die Sparkasse hat die fristlose Kündigung der Darlehensschuldner auch nicht zurückgewiesen, zumal diese den Kredit aufgrund des Darlehensvertrags mit der Westdeutschen Landesbank auch tilgen konnten. Sie hat nur eine Vorfälligkeitsentschädigung4 verlangt, d.h. eine Entschädigung wegen des ihr durch vorzeitige Aufhebung entgehenden (Zins-)Gewinns. Die Banken halten im Allgemeinen ein zur fristlosen Kündigung des Kunden berechtigendes eigenes Verschulden nicht für möglich und regeln es deswegen von vornherein nicht gesondert in ihren AGB.

\footnotetext{
3 Wie in jedem Dauerschuldverhältnis besteht auch beim Darlehen ein zwingendes Recht zur außerordentlichen Kündigung, s. nur BGHZ 29, I72; 95, 372.

4 Siehe dazu BGHZ i36, I6I; BGH, NJW 2001, 509.
} 
Nun könnte man natürlich den Versuch einer ergänzenden Vertragsauslegung dahin

starten, dass der Kunde in jedem Fall vorzeitiger Darlehensauflösung eine Vorfälligkeitsentschädigung zu begleichen hat. Für den Fall eines eigenen schuldhaften Verhaltens der Bank würde aber eine ausdrückliche Vertragsklausel dieser Art an der AGB-rechtlichen Inhaltskontrolle scheitern, da Verschulden der Bank nicht zu einer Sanktion für den Kunden führen darf. Dementsprechend kann der Vertrag auch nicht in diese Richtung ergänzend ausgelegt werden. War die fristlose Kündigung der Darlehensschuldner Domrath also wirksam, so mussten sie keine Vorfälligkeitsentschädigung bezahlen. ${ }^{5}$

Sollte die fristlose Kündigung dagegen nicht wirksam gewesen sein, durfte die Sparkasse sie aber ebenfalls nicht verlangen, da dann der Darlehensvertrag einfach weiterlief. Die Sparkasse selbst hat nicht gekündigt, sie hat dem Schuldner nur die Auflösung gegen Vorfälligkeitsentschädigung angeboten, was dieser aber stets abgelehnt hat. Im Falle unberechtigter fristloser Kündigung des Kunden war mangels Aufhebungsvertrags der Darlehensvertrag also keineswegs beendet. Der Darlehensrückzahlungsanspruch konnte nur fällig werden, wenn die fristlose Kündigung des Schuldners berechtigt war. In keinem Fall - das steht auch ohne weitere Sachverhaltsaufklärung fest - schuldeten also die Eheleute Domrath eine Vorfälligkeitsentschädigung.

\section{d) Der Annahmeverzug der Bank}

Denkbar ist ferner, dass die Eheleute Domrath durch späteres Verhalten eine Vertragsverletzung begangen haben, insbesondere durch Nichtzahlung von Zins und Tilgung, nachdem die Sparkasse die Umschuldung mit Hilfe der Westdeutschen Landesbank wegen mangelnder Vorfälligkeitsentschädigung abgelehnt hatte. Insoweit ist jedoch $\ 30$ I BGB heranzuziehen. Diese Vorschrift befreit den Schuldner während des Annahmeverzuges des Gläubigers endgültig von jeder Zinspflicht, ${ }^{6}$ so dass die Sparkasse, als sie die Annahme der ausgekehrten Darlehensvaluta von 230.000 DM verweigert hatte, auch nicht die vertraglichen $6 \%$ Zins pro Jahr fordern konnte. Um Gläubigerverzug herbeizuführen, bedurfte es gem. \294 BGB eines tatsächlichen Angebots durch den Schuldner,7 während gem. \295 BGB ein wörtliches Angebot genügte, wenn der Gläubiger erklärt hatte, die Leistung (etwa mangels gleichzeitiger Vorfälligkeitsentschädigung) nicht annehmen zu wollen. ${ }^{8}$ Zumindest solange die Westdeutsche Landesbank also die geschuldeten 230.000 DM nebst Zinsen (soweit sie bis zum Eintritt des Gläubigerverzugs fällig waren) bereit hielt, bestand der Gläubigerverzug fort. So war es in jedem Fall, solange die Darlehensschuldner durch Zahlung von Bereitstellungszinsen für die jederzeitige Abrufbarkeit der Summe sorgten. Der Annahmeverzug kann enden, wenn eine seiner Voraussetzungen (etwa das Angebot) entfällt. ${ }^{9}$ Dass ein Schuldner das Angebot aber auch dann aufrecht erhalten muss, wenn der Gläubiger die Annahme endgültig abgelehnt hat, ist zu verneinen. ${ }^{1 \circ}$ Die Sparkasse ist aber weder auf das Angebot zur Umschuldung je eingegangen, noch hat sie wegen Nichtzahlung von Zins und Tilgung eine Kündigung ausgesprochen, sondern sich nur bei der Verteilung des Versteigerungserlöses hinsichtlich ihrer Zinsforderung Befriedigung verschafft. Das ist zudem in der Weise

\footnotetext{
s Bei wirksamer fristloser Kündigung wegen vertragswidrigen Verhaltens schuldet umgekehrt die gekündigte Bank Schadensersatz, vgl. Palandt/Putzo, BGB, 6I. Aufl., $\$ 609$ Rn. I 8, zum alten Recht, das hierauf anzuwenden war.

6 Siehe nur Palandt/Heinrichs (o. Fn. 5), \30I Rn. I.

7 Siehe dazu BGHZ 90, 359.

$8 \mathrm{Zu}$ den Anforderungen an die Verweigerungserklärung s. BGH, ZIP 1999, 445.

9 Siehe nur Palandt/Heinrichs (Fn. 5), \293 Rn. I 2.

Io BGH, NJW 200I, 287.
} 
geschehen, als ob der Darlehensvertrag bis zur Befriedigung weitergeführt worden wäre, also mit Zinsen und zusätzlichem Verzugsschadensersatz. Insofern hat die Sparkasse bei ihrer Abrechnung also die Kündigung des Darlehensnehmers im Gegensatz zu ihrem früheren Verhalten nicht mehr zugrunde gelegt.

\section{Das Zwangsversteigerungsverfahren}

\section{a) Die Vollstreckung wegen unberechtigter Forderungen}

Die Sparkasse machte also einen Anspruch auf Vorfälligkeitsentschädigung in Höhe von $3.000 \mathrm{DM}$ geltend, der weder nach Vertrag noch nach Gesetz begründet war. Diesen Anspruch hätte sie einklagen und sich im Übrigen mit der Darlehensrückzahlung begnügen können. Dazu hatte sie jedoch keine Neigung, sondern leitete das Zwangsversteigerungsverfahren ein, das nach vielen Jahren zu ihrer vollen Befriedigung führte, wenn auch ohne die Vorfälligkeitsentschädigung. Ex post betrachtet kann man also sagen, dass das Vollstreckungsverfahren wegen nichts und wieder nichts durchgeführt worden ist. Dass der Schuldner Domrath zur Sicherung der Durchführung eines solchen rechtsmissbräuchlichen Verfahrens für prozessunfähig erklärt werden musste, ist die traurige Pointe.

Wie ist so etwas möglich, wird nicht nur der Laie fragen. Das ergibt sich, wie angesprochen, aus der Vollstreckungsmacht der Banken aufgrund der von ihnen von vornherein eingeholten vollstreckbaren Urkunden, mit denen sich der Schuldner bei Abschluss des Darlehensvertrags gem. $\$ 794$ Abs. I Nr. 5 ZPO der sofortigen Zwangsvollstreckung wegen des gesamten Rückzahlungsanspruchs nebst Zinsen unterwerfen muss. ${ }^{\text {II }}$ Vollstreckungsgegenstand war dann auch die notarielle Grundschuldbestellungsurkunde der Eheleute Domrath vom Mai 1985 , aufgrund deren die Sparkasse aus der ihr bestellten Grundschuld 350.000 DM nebst i 8\% Zinsen seit Eintragung im Grundbuch sowie die Kosten der Rechtsverfolgung verlangen konnte. Auf Antrag der Sparkasse wurde dementsprechend das Versteigerungsverfahren angeordnet, das keine materielle Anspruchsprüfung einschließt. Voraussetzung ist vollstreckungsrechtlich allerdings die Erteilung einer Vollstreckungsklausel.

\section{b) Die Erteilung der Vollstreckungsklausel}

Die Zwangsvollstreckung wird bei Urteilen gem. \724 Abs. I ZPO aufgrund einer mit der Vollstreckungsklausel versehenen Ausfertigung (vollstreckbare Ausfertigung) durchgeführt. Die Vollstreckungsklausel, die die Vollstreckungsorgane entlastet und bindet, wird auf eine Ausfertigung des Urteils gesetzt und lautet dahin, dass »Vorstehende Ausfertigung dem... zum Zwecke der Zwangsvollstreckung erteilt wird « ( $(725$ ZPO). Zuständig ist in der Regel der Urkundsbeamte der Geschäftsstelle des Gerichts des ersten Rechtszuges, das über den Rechtsstreit entschieden hat. Auf vollstreckbare Urkunden sind die Regeln über die Zwangsvollstreckung aus Urteilen gem. $\$ 795$ ZPO nur zum Teil entsprechend anwendbar. Die Vollstreckung aus vollstreckbaren Urkunden richtet sich primär nach $\ 797$ ZPO. ${ }^{12}$ Die vollstreckbare Ausfertigung einer notariellen Urkunde wird gem. \802 ZPO ausschließlich von dem Notar erteilt, der die Urkunde verwahrt (\797 Abs. 2 ZPO). Die Entscheidung über

I I Siehe zum System der Titulierung nur Baur/Stürner, Sachenrecht, 17. Aufl., $\ 37$ Rn. 55 und $\ 40$ Rn. $36 \mathrm{ff}$.

I 2 Bei der Vollstreckung werden insbesondere nicht mehr das Bestehen des Anspruchs und seine Fälligkeit geprüft, s. dazu nur Thomas/Putzo, ZPO, 24. Aufl., $\$ 797$ Rn. I. 
Einwendungen, die die Zulässigkeit der Vollstreckungsklausel betreffen, trifft das

Amtsgericht, in dessen Bezirk der Notar seinen Amtssitz hat ( $\$ 797$ Abs. 3 ZPO).

Dem Schuldner stehen dabei drei Rechtsbehelfe zur Verfügung, die Klauselerinnerung nach $\ 732 \mathrm{ZPO}$, die Vollstreckungsgegenklage nach $\ 767 \mathrm{ZPO}^{13}$ und die Klauselgegenklage nach $\$ 768 \mathrm{ZPO} .^{14}$

\section{c) Der Rechtsschutz gegen die Klauselerteilung}

Der Notar, der die vollstreckbare Grundschuldbestellungsurkunde zugunsten der Stadtsparkasse Hilden errichtet hatte, konnte also auch die vollstreckbare Ausfertigung unschwer erteilen, um die Zwangsvollstreckung zu ermöglichen. Dabei war jedoch zu beachten, dass nach den Vertragsbedingungen der Anspruch aus der

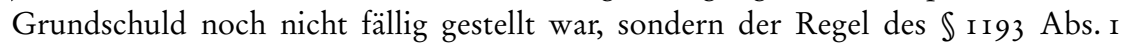
Satz I BGB entsprechend erst nach vorgängiger Kündigung fällig werden sollte. ${ }^{\text {Is }}$ Demgemäß ergab sich die Fälligkeit des Grundschuldanspruchs nicht aus der vollstreckbaren Urkunde. Für diesen Fall sieht $\$ 726$ Abs. I ZPO vor, dass ein Nachweis durch öffentliche oder öffentlich beglaubigte Urkunden geführt werden muss. ${ }^{16}$

Um diesen Beweis nicht führen zu müssen, ist es weithin üblich, in der vollstreckbaren Urkunde den Schuldner bereits von vornherein auf den Nachweis der Entstehung des Anspruchs und seiner Fälligkeit verzichten zu lassen. Das hat die Rechtsprechung, obwohl eine Inhaltskontrolle solcher Formularklauseln nach dem AGB-Gesetz möglich war, immer wieder gebilligt. Lapidar hat der BGH Anfang der 8oer Jahre diese Praxis akzeptiert. ${ }^{17}$ Der Gläubiger werde durch die Vereinbarung, dass er »jederzeit ohne Nachweis des Entstehens und der Fälligkeit der Zahlungsverpflichtungen« eine vollstreckbare Ausfertigung erhalten könne, von einem solchen Nachweis wirksam entbunden. Es bestehe kein sachlicher Grund, es dem Schuldner zu »verwehren«, auf den Schutz des $\$ 726$ Abs. I ZPO zu verzichten. Das gelte auch für die Tatsache der Hingabe des Darlehens. Diese Rechtsprechung hat auch das BayObLG übernommen, ${ }^{18}$ obwohl es eine AGB-rechtliche Inhaltskontrolle durchgeführt hat. Die Argumentation dafür stellte lediglich auf das Interesse der Banken als Immobiliargläubiger an einer unmittelbaren Vollstreckung ab. Sie erhalten also praktisch automatisch neben dem Titel somit auch die Vollstreckungsklausel. Die Klauselerinnerung des Schuldners nach $\$ 732$ ZPO, mit der formelle Rügen gegen die Zulässigkeit der Vollstreckungsklausel geltend gemacht werden können (etwa wegen fehlenden Urkundennachweises), und die Klauselgegenklage nach $\ 768$ ZPO, mit der auch materielle Einwände (etwa wegen des Fehlens einer Kündigung als Bedingung für den Eintritt der Fälligkeit nach $\ 726$ Abs. I ZPO) ${ }^{19}$ erhoben werden können, werden dem Schuldner somit von vornherein genommen. Er hat dann lediglich die Möglichkeit, gem. \$S 795, 797 Abs. 4, 767 ZPO Vollstreckungsgegenklage zu erheben. Die Verfahren nach den $\$ \$ 732,768$ ZPO werden als Rechtsbehelfe des Schuldners also ausgeschaltet.

I 3 Ohne die Präklusion von Einwendungen nach $\$ 767$ Abs. 2 ZPO, wie $\$ 797$ Abs. 4 ZPO anordnet.

I4 Siehe nur Musielak/Lackmann, ZPO, 3. Aufl., $\$ 768$ Rn. 9 zur Trias der Rechtsbehelfe.

Is Nach der gesetzlichen Regel des $\$ I I93 Abs. I Satz 3 BGB mit sechsmonatiger Frist.

I6 Da die Vollstreckung nach dem Inhalt der Urkunde von dem durch den Gläubiger zu beweisenden Eintritt einer anderen Tatsache als einer dem Gläubiger obliegenden Sicherheitsleistung abhängt, greift $\$ 726 \mathrm{Abs}$. I ZPO ein. Er findet insbesondere Anwendung, wenn eine Kündigung des Gläubigers erforderlich ist (OLG Stuttgart, NJW-RR 1986, 549), wobei deren Zugang zu beweisen ist.

I7 BGH, NJW I98I, 2756, 2757.

I 8 BayObLG, NJW-RR I995, 782; ebenso Rastätter, NJW I991, 392.

I9 Siehe nur Musielak/Lackmann (Fn. I4), $\$ 768$ Rn. I. 
Mit einer solchen Vollstreckungsgegenklage hätten die Darlehensnehmer geltend machen können, dass es sich bei der Grundschuld um eine Sicherheit für den in Höhe von 230.000 DM ausgezahlten Kredit handelte, die beanspruchte Vorfälligkeitsentschädigung davon nicht gedeckt war, die Sparkasse mit der Annahme der Valuta nach fristloser Kündigung im Gläubigerverzug war und deswegen gem. \30I BGB seither auch keine Zinsen mehr zu beanspruchen hatte. Damit wäre nachträglicher Rechtsschutz gegen das missbräuchliche Vorgehen der Sparkasse möglich gewesen.

Die Eheleute Domrath sind diesen Weg jedoch - eine lebenspraktisch unheilvolle Entscheidung - nicht gegangen, sondern haben sich von Anfang an geweigert, selbst Klage zu erheben und für Anwalts- und Gerichtskosten insoweit aufzukommen. Sie sahen nicht ein, warum sie klagen sollten, nachdem das Kreditinstitut zu Unrecht eine Forderung gegen sie erhoben hatte. Es ist auch verstehbar, dass sie sich mit der Vollstreckungsgegenklage nicht abfinden wollten. In den maßgeblichen Jahren galten noch die Rechtsgrundsätze des III. Zivilsenats des BGH vom Anfang der 8oer Jahre, ${ }^{20}$ nach der der Schuldner bei der Vollstreckungsgegenklage gegen eine vollstreckbare Urkunde die Beweislast für das Nichtbestehen der Forderung hatte. Das war zwar nicht ohne Kritik geblieben, ${ }^{21}$ ist in der Praxis aber weithin zugrunde gelegt worden. Der XI. Zivilsenat des BGH hat erst im Jahre 200I die Unerträglichkeit dieser Beweislastverteilung erkannt und dem Gläubiger die Beweislast für die Hingabe eines Darlehens auferlegt, auch wenn sich der Schuldner der sofortigen Zwangsvollstreckung unterworfen und auf den Nachweis der Fälligkeit verzichtet hat. ${ }^{22}$ Die Gründe dafür sind zwingend. Unabhängig von der Parteirolle im Prozess muss der Gläubiger einer Forderung die Voraussetzung seines Rechts darlegen und beweisen. Sonst käme es zu nicht hinnehmbaren Widersprüchen zwischen Prozessen mit Feststellungsklage des Gläubigers und Vollstreckungsabwehrklage des Schuldners. Die notarielle Vollstreckungsunterwerfung ist nur eine einseitige prozessuale Erklärung, die keine materiellrechtlichen Auswirkungen haben kann. Mangels Rechtskraftwirkung der vollstreckbaren Urkunde kann auch der Angriff auf sie nicht beschränkt sein. Das kann auch nicht aus der Klausel mit dem Verzicht auf den Fälligkeitsnachweis hergeleitet werden, da diese nach dem XI. Zivilsenat nur der Vereinfachung, nicht der Beweislastumkehr dient. Schwierigkeiten bleiben auch nach dem neuen Urteil des BGH weiterhin insoweit, als sich die Kreditinstitute ein kausales oder abstraktes Schuldanerkenntnis geben lassen, das zu einer Umkehr der Beweislast führen kann. ${ }^{23}$

Von der früheren Rechtsprechung aus mussten die Eheleute Domrath bei Erhebung der Vollstreckungsgegenklage damit rechnen, das gesamte Vertragsverhältnis mit der Stadtsparkasse Hilden einschließlich der Vertragsstörungen im Zusammenhang mit dem Hausbau und dem Bauträgergeschäft bis hin zu dem Umschuldungsangebot

20 BGH, NJW I98I, 2756.

2 I Auch die obergerichtliche Rechtsprechung verweigerte teilweise den Gehorsam, s. OLG Nürnberg, DNotZ 1990, 564, 565; OLG Celle, NJW-RR 1991, 667; OLG Hamm, DNotZ 1994, 57, 59; OLG Düsseldorf, NJW-RR I 997, 444; aus dem kritischen Schrifttum ist hervorzuheben: Wolfsteiner, NJW I982, 285 I; Münch, NJW I991, 795, 796; Baumgärtel, in: Festschrift für Lüke, S. I, 4; Stein/Jonas/Münzberg, ZPO, 2 I. Aufl., $\$ 767$ Rn. 44; dagegen ist das OLG München dem III. Zivilsenat gefolgt (NJW-RR I992, I 25); ebenso für den Fall des Verzichts auf den Nachweis der Fälligkeit bei der Klauselerteilung Schuschke, in: Schuschke/Walter, Vollstreckung und vorläufiger Rechtsschutz, Bd.I, 2. Aufl., $\$ 767$ ZPO Rn. 38 ; Zöller/Herget, ZPO, 22. Aufl., $\$ 767$ Rn. I I.

22 BGH, NJW $2001,2096$.

23 In dem entschiedenen Fall wurde ein solches kausales Schuldanerkenntnis im Wege der Interpretation ausgeschlossen. 
darlegen und beweisen zu müssen. Dass sie davor zurückgeschreckt sind, ist verständlich, auch wenn - wie gesagt - ein pragmatischer Rat ihnen den Rechtsschutz über die Vollstreckungsgegenklage zur Vermeidung existentieller Nachteile ans Herz legen musste. Wahrscheinlich wäre das auch gelungen, wenn ihnen bei dem entsprechenden Hinweis nicht immer die »Rechtslage « vorgehalten, sondern die Machtlage erklärt und eine realistische Reaktion darauf empfohlen worden wäre.

Insgesamt blieb es somit dabei, dass das Vollstreckungsgericht bis zum Schluss auf seine mangelnde Zuständigkeit für die Beurteilung der materiellrechtlichen Ansprüche verwiesen, das Prozessgericht aber mangels Vollstreckungsgegenklage niemals eine Überprüfung vorgenommen hat. Sämtliche vollstreckungsrechtlichen Rechtsbehelfe blieben dann auch erfolglos, selbst die ultima ratio des Vollstreckungsschutzantrags nach $\ 765$ a ZPO wegen sittenwidriger Härte. Auch insofern wurde den Darlehensschuldnern entgegengehalten, die Vollstreckungsmaßnahme als solche müsse eine sittenwidrige Härte darstellen, ${ }^{24}$ während Einwände gegen den Titel vorher (mit der Vollstreckungsgegenklage) abzuwehren seien. Karl Wilhelm Domrath ist dementsprechend, obwohl er materiellrechtlich im Recht war, immer mehr in vollstreckungsrechtliches Gestrüpp geraten, auch soweit dieses - wie die erwähnte Rechtsprechung des III. Zivilsenats des BGH - dringend bis zur Wurzel hätte abgeschnitten werden müssen.

Alle Eingaben und Verfassungsbeschwerden haben sich ebenso als erfolglos erwiesen, weil die Spitzen der Legislative, Exekutive und Judikative gar nicht in der Lage und willens sind, in ein hochdifferenziertes institutionalisiertes, wirtschaftliches und rechtliches System wie das des Immobiliarkredits gezielt zu intervenieren oder auch nur eine Detailanalyse seiner Defizite zu realisieren. Der Existenzverlust eines Einzelnen löst meist noch nicht einmal die Selbstkritik eines Funktionsträgers aus. Der Fall der Domraths macht aber deutlich, dass es einer Gesamtüberprüfung des Vollstreckungszugriffs der Banken und der Entwicklung eines Alternativkonzepts bedarf, das wirtschaftlich tragbar ist und ein individualrechtliches Schutzminimum gewährleistet.

\section{Alternativkonzepte}

Die formularmäßige Ausgestaltung der Vertragsurkunden von Immobiliarkreditgebern, die eine Vollstreckungsunterwerfung fordern, ist ein »kautelarjuristisches Kunstwerk «, wie ihr bislang entschiedenster, aber resignierender Kritiker ${ }^{25}$ es formuliert hat. Angriffspunkte bietet es deswegen mannigfach, auch wenn heute bei einem derartigen langfristigen Geschäft nur noch wenige mit Aussicht auf Erfolg geltend gemacht werden können. In Betracht kommen theoretisch eine AGB-rechtliche Prüfung, die sich auf das dem Darlehensnehmer abverlangte kausale oder abstrakte Schuldanerkenntnis beziehen kann, eine Ächtung überhöhter Grundschuldzinsen nach $\int I_{3} 8 \mathrm{BGB}$, eine Inhaltskontrolle der dinglichen und persönlichen Unterwerfung unter die sofortige Zwangsvollstreckung, eine Verwerfung der Klausel über den Verzicht auf den Nachweis der Fälligkeit der Grundschuld, eine Rechtsmissbrauchskontrolle bei der Klauselerteilung und den Entscheidungen der Vollstreckungsgerichte bis hin zum Vollstreckungsschutz nach $\$ 765$ a ZPO. Da diese Ebenen hier nicht mit dem Anspruch auf eine präzise rechtsdogmatische Alternative behandelt werden 
können, sollen erst einmal die Vorgaben der zentralen Entscheidungen des BGH zu diesem Sektor zugrunde gelegt werden.

Maßgeblich ist in erster Linie ein Urteil aus dem Jahre $1986,{ }^{26}$ das erstmals eine ernsthafte AGB-rechtliche Prüfung unternommen hat. Inzwischen ist die Inhaltskontrolle notarieller Verträge sogar erweitert. \310 Abs. 3 Nr. I BGB sieht bei Verbraucherverträgen in Anknüpfung an $\ 24$ a AGBG vor, dass AGB als vom Unternehmer gestellt angesehen werden, es sei denn, dass sie durch den Verbraucher in den Vertrag eingeführt wurden. Diese Norm ist deswegen auch dann anwendbar, wenn die formularmäßige Regelung auf Vorschlag eines Notars Vertragsinhalt geworden ist. ${ }^{27}$ Damit ist der Pauschaleinwand entkräftet, der Notar sorge schon für ausgewogene Verträge, was im Hinblick auf die den Bankenwünschen folgenden Vertragsmustersammlungen der Notare nie zutraf.

Der BGH hat nun 1986 zunächst die Vollstreckungsunterwerfung des Schuldners als solche gebilligt, da diese von der ZPO gestattet sei, sie die Durchsetzung des gesicherten Anspruchs erleichtere und der Schutz des Schuldners durch das Erfordernis notarieller Beurkundung und die Vollstreckungsrechtsbehelfe, insbesondere auch die Vollstreckungsabwehrklage, in ausgewogener Weise gewährleistet sei. Es sei nicht das Leitbild des Gesetzes, dass eine Zwangsvollstreckung erst nach einem gerichtlichen Erkenntnisverfahren stattfinde. Die Unterwerfungsklausel als solche stelle danach keine unangemessene Benachteilung des Schuldners im Sinne des AGBRechts dar. Die Banken als Immobiliarkreditgeber brauchen deswegen, anders als die meisten Gläubiger, ihren Schuldnern nicht mit einem Klagverfahren hinterherzulaufen. Die Rechtfertigung dieses Privileges mit den Gefahren der Vermögensverschlechterung beim Schuldner ist zwar beim erstrangigen Kredit äußerst fragwürdig. Die Rechtfertigung mit den (möglichen) Komplikationen des Verwertungsverfahrens ist aber eher tragfähig. Das Ergebnis des BGH ist jedenfalls insoweit akzeptierbar.

Das formularmäßige abstrakte Schuldanerkenntnis in Höhe des Grundschuldbetrags, das der Schuldner meist abzugeben hat und das mit der Unterwerfung unter die sofortige Zwangsvollstreckung in das sonstige persönliche Vermögen garniert wird, war nicht nur anhand der Generalklausel des AGB-Rechts zu prüfen, sondern auch anhand des $\$ I I Nr. I s AGBG (heute $\ 309$ Nr. I 2 BGB), da ein solches Anerkenntnis die Beweislast wegen erbrachter Leistungen verschiebt. Hierzu hat der BGH I986 angeführt, die Vereinbarung einer abstrakten Zahlungsverpflichtung des Schuldners als Sicherheit für eine Kreditvereinbarung falle nicht unter $\$ I I Nr. Is AGBG, auch wenn sie zu einer für den Schuldner ungünstigen Beweislastverteilung führe. Auch dafür wurden die schutzwürdigen Gläubigerinteressen angeführt. I99I hat der XI. Zivilsenat dies wenigstens dadurch gemildert, dass er die formularmäßige persönliche Haftungsübernahme und Vollstreckungsunterwerfung Dritter, die eine Grundschuld als Sicherheit für die Kreditverbindlichkeit des Darlehensschuldners zur Verfügung stellen, nach $\$ 9$ AGBG wegen unangemessener Haftungserweiterung für unwirksam erklärt hat ${ }^{28}$ Es bleibt aber dabei, dass ein Schuldanerkenntnis in Höhe des Grundschuldbetrags mit Vollstreckungsunterwerfung weiterhin bei der Grundschuldbestellung vom Darlehensschuldner verlangt werden kann, wenn man die Rechtsprechung des BGH zugrunde legt.

Dass sich der Schuldner der sofortigen Zwangsvollstreckung unterwirft, bedeutet keine zusätzliche strukturelle Belastung; dass er dies auch hinsichtlich seines sonstigen Vermögens neben dem Grundstück tun muss, lässt sich noch damit rechtfertigen, dass 
dem Darlehensgläubiger, der die sofortige Vollstreckung in das Grundstück betreibt, nicht daneben ein gerichtliches Erkenntnisverfahren (möglicherweise über drei Instanzen bis zur Rechtskraft) abverlangt werden kann. Problematisch bleibt aber vor allem die Beweislastverschiebung aufgrund des abstrakten Schuldanerkenntnisses, wenn damit dem Schuldner der Beweis hinsichtlich anspruchsbegründender Voraussetzungen auch gegenüber einer vollstreckbaren Urkunde zugemutet wird. Das widerspricht zumindest der Tendenz der Entscheidung des XI. Zivilsenats aus dem Jahre 200I. ${ }^{29}$ Diese Beweislastverteilung wirkt sich nicht im Klauselerteilungsverfahren, sondern bei der Vollstreckungsabwehrklage als dem Hauptbehelf des Schuldners aus und verkürzt damit seinen Rechtsschutz. Der Schuldner soll gegenüber dem abstrakten Schuldanerkenntnis nach $\$ 78$ I BGB nur noch die Bereicherungseinrede des $\int 82$ I BGB haben und dafür die maßgeblichen Tatsachen dartun, also von der Darlehensauszahlung bis zur Verrechnung der einzelnen Leistungen auf Kapital, Zinsen und Nebenleistungen. Damit wird eine ausgewogene Gesamtregelung verfehlt. Insofern bedarf es einer rechtsdogmatischen Korrektur auf der Basis der Generalklausel des $\ 307$ BGB. Diese muss zum Ergebnis haben, dass ein Schuldanerkenntnis zumindest hinsichtlich der anspruchsbegründenden Voraussetzungen, etwa der Darlehensauszahlung, unwirksam ist. Das ist den Banken auch zumutbar, da sie insoweit durch öffentliche Urkunden gem. \4 8 Z ZPO, insbesondere notarielle Urkunden, Vorsorge treffen können. Die Stadtsparkasse Hilden hätte somit a limine nicht wegen mehr als 230.000 DM vorgehen können, da sie den Rest nicht ausgezahlt hatte.

Desgleichen bedarf es einer AGB-rechtlichen Inhaltskontrolle der Nachweisverzichtsklausel, soweit den Kreditinstituten ein Nachweis der Vollstreckungsvoraussetzungen für die Klauselerteilung durch öffentliche oder öffentlich beglaubigte Urkunden gem. $\$ 726$ Abs. I ZPO zugemutet werden kann. Das gilt wiederum für die anspruchsbegründenden Tatbestandsmerkmale. Verlangt man etwa den Nachweis einer Kündigung durch öffentliche Urkunde, dann werden die Kreditinstitute allerdings wahrscheinlich offensiv reagieren und die Grundschuld, wie schon verschiedentlich vorgeschlagen, von Anfang an fällig stellen, so dass es einer Kündigung gar nicht mehr bedarf. Damit könnte, wenn dies zugelassen wird, die gerichtliche Überprüfung vollends auf die Vollstreckungsgerichte verschoben werden, an die aber gleichfalls neue Anforderungen zu stellen sind.

Den Vollstreckungsgerichten muss im Hinblick auf die von den Banken aufgebaute Zugriffsmacht ein Minimum an Rechtsmissbrauchskontrolle auferlegt werden, was im Hinblick auf die anerkannte Geltung des $\ 242$ BGB auch im Prozessrecht ohne weiteres möglich ist. Ein willkürlicher Vollstreckungsantrag ist grundsätzlich zurückzuweisen. Um einen solchen handelt es sich etwa, wenn ein Kreditinstitut nach kürzester Vertragslaufzeit einfach ohne plausible Begründung Versteigerungsantrag stellt oder nach langjähriger Vertragsabwicklung ohne jede Abrechnung der erbrachten Leistungen und Kündigung vorgeht. Damit ist zwar nur eine Evidenzkontrolle einzurichten, die aber immerhin ausschließt, dass wegen jeden beliebigen wirtschaftlichen Dissenses der Parteien Vollstreckungmacht ausgeübt werden kann. Im Fall der Eheleute Domrath hätte das Vollstreckungsgericht bei der bloßen Begründung des Versteigerungsantrags mit dem ohne Vertragsgrundlage erhobenen Anspruch auf Vorfälligkeitsentschädigung danach dessen Nachweis durch ein Urteil verlangen müssen. Insofern wäre das Vollstreckungsgegenklageverfahren, bei dem der Schuldner Klagrisiko und Kostenaufwand übernehmen muss, in sachgemäßer Weise zu entlasten. Ferner ist auch im Vollstreckungsgegenklageverfahren zu sichern, dass der 
Schuldner nicht die anspruchsbegründenden Tatbestandsvoraussetzungen des geltend gemachten Anspruchs beweisen muss, auch nicht aufgrund eines Schuldanerkenntnisses. Schließlich kann sich ein Rechtsmissbrauch auch noch bis zum Schluss der Vollstreckung herausstellen und ist dann auch bei Vollstreckungsschutzanträgen nach $\$ 765$ a ZPO zu beachten. Außerdem ist bei der Verteilung des Versteigerungserlöses eine intensivere gerichtliche Kontrolle der geltend gemachten Ansprüche nötig, was die Abrechnung insbesondere der Zinsen und des Verzugsschadens angeht. Die Stadtsparkasse Hilden hätte dann die Abrechnung mit der Vorfälligkeitsentschädigung nicht einfach durch die Abrechnung mit (wegen \30I BGB zumindest teilweise nicht geschuldetem) laufendem Vertragszins und Verzugsschadensersatz bis zur Befriedigung austauschen können.

\section{Konsequenzen}

Es versteht sich, dass die hier vorgeschlagenen Alternativen eine minutiöse rechtsdogmatische Präzisierung erfordern, um bislang herrschende Interpretationen vor allem vollstreckungsrechtlicher Normen zurückdrängen zu können. Der radikalere Vorschlag, das gerichtliche Erkenntnisverfahren mit seiner materiellen Rechtskontrolle zu Lasten des Vollstreckungsverfahrens stärker zur Geltung zu bringen, ist als gescheitert anzusehen. In jedem Fall wirft die von den Banken entwickelte Vollstreckungsmacht beim Grundpfandkredit ein ganzes Kompendium an Rechtsfragen auf, wenn ein erträglicher Individualrechtsschutz gesichert werden soll. Karl Wilhelm Domrath ist daran verzweifelt und von einer ebenso überempfindlichen wie zu Selbstkritik unfähigen nordrhein-westfälischen Provinzjustiz akkurat solange für prozessunfähig erklärt worden, bis das Vollstreckungsverfahren beendet war. Sein Fall sollte wenigsten geeignet sein, Anwälten und Gerichten, die eine ausgewogene Rechtsordnung auch in diesem Bereich für möglich halten, die Augen für die notwendigen Restriktionen der Vollstreckungsmacht der Banken zu öffnen. 